\title{
Escala para la calidad de los servicios y lealtad del consumidor en tiendas de alimentación*
}

\author{
Scale for Service Quality and Customer Loyalty in Groceries
}

Recibido: junio 15 de 2013 | Revisado: marzo 20 de 2014 | Aceptado: marzo 27 de 2014

\author{
AlBERT FORNIELES ** \\ EVA PENELo \\ GASPAR BERBEL \\ REMEI PRAT \\ Universitat Autònoma de Barcelona, España
}

doi:10.11144/Javeriana.UPSY13-3.ecsl

Para citar este artículo: Fornieles. A., Penelo, E., Berbel, G. \& Prat, R. (2014). Escala para la calidad de los servicios y lealtad del consumidor en tiendas de alimentación. Universitas Psychologica, 13(3), 985-994. http://dx.doi.org/10.11144/Javeriana.UPSY13-3.ecs

Artículo original resultado de investigación.

*** Universitat Autònoma de Barcelona, España. Departament de Psicobiologia i Metodologia de les Ciències de la Salut. Correos electrónicos: albert. fornieles@uab.cat,eva.penelo@uab.cat,gaspar. berbel@uab.cat, remei.prat@uab.cat

\section{RES U MEN}

Actualmente el comercio local está amenazado por la competencia de las grandes superficies, siendo crucial apostar por la satisfacción y lealtad. El objetivo del estudio fue elaborar un instrumento para evaluar la satisfacción y la lealtad en tiendas de alimentación y estudiar la influencia de variables sociodemográficas. Se aplicó una entrevista personal compuesta por 36 ítems agrupados en seis dimensiones: trato-clientela, instalaciones, producto, servicios, fidelidad y valor añadido, a 712 participantes. Las puntuaciones globales fueron positivas y homogéneas, destacando las escalas de producto, lealtad y trato-clientela, y siendo este último el factor más explicativo de la satisfacción. Los hombres y el grupo de 21-35 años presentan una menor satisfacción. Tres escalas se asocian positivamente con la escala de lealtad. Palabras clave

calidad del servicio; comercio local; cuestionario; lealtad; satisfacción del cliente

\footnotetext{
A B S T R A C T

Local trade is currently under the threat of competition from department stores; thus, it is crucial to concentrate on satisfaction and loyalty. To develop an instrument to evaluate satisfaction and loyalty in delicatessens and to study the influence of socio-demographic variables on these aspects. A personal interview was applied that was composed of 36 items grouped into six dimensions: treatment of costumers, facilities, product, services, loyalty and value added, conducted with 712 participants. Positive and homogenous overall scores were obtained, specially product, loyalty and handling of customer scales, the latter being the most explicative factor of satisfaction. Men and the 21-35 year age group show low satisfaction. Three scales are positively associated with the loyalty scale.

Keywords

quality of service; local trade; questionnaire; loyalty; customer satisfaction
} 


\section{Introducción}

Sin duda, uno de los principales motores económicos del país es el comercio local, con una oferta que permite dinamizar barrios y ciudades. El ritmo impuesto por el modo de vida actual complica acudir a diferentes tiendas especializadas, por lo que el comercio local está decayendo en favor de las grandes superficies y otras modalidades de oferta más periféricas, que facilitan el proceso de compra al agrupar muchos productos de tipología muy diversas, y que ofrecen, en muchas ocasiones, la ventaja de un menor coste del producto. Este hecho se acentúa en tiempos de crisis, cuando el coste económico de los productos se vuelve más importante. En este escenario altamente competitivo, la apuesta por la calidad supone una cuestión crucial, al ser la percepción de dicha calidad uno de los atributos principales de la satisfacción. A su vez, se ha de tener en gran consideración la satisfacción de los clientes, ya que esta probablemente influirá de forma importante en su lealtad.

En las dos últimas décadas, ha habido numerosas iniciativas psicométricas para medir la satisfacción de los clientes. Johnson, Herrmann y Gustafsson (2002) destacan los denominados índices nacionales, como el Swedish Customer Satisfaction Barometer (SCSB), el American Customer Satisfaction Index (ACSI) o el European Customer Satisfaction Index (ECSI). Además, el ECSI incorpora tres ítems para medir la lealtad de los clientes, preguntando sobre la intención de volver a comprar y de recomendar el establecimiento o producto a otras personas.

No obstante, la metodología más empleada es el SERVQUAL (Parasuraman, Zeithaml \& Berry, 1988, 1991), que evalúa la "calidad" de un servicio como la diferencia entre el servicio recibido y las expectativas que se espera obtener de dicho servicio. Dicha metodología se centra en tres grandes dimensiones, bloques o requisitos: servicio (cortesía, credibilidad, capacidad de respuesta, comprensión del cliente, fiabilidad, comunicación, seguridad y accesibilidad), elementos tangibles (instalaciones, equipos y mobiliario, envases y embalajes, documentos de compra y productos) y personal (imagen y competencia profesional). En el sector de la hostelería y restauración son numerosos los estudios que han empleado y/o adaptado esta metodología (Andaleeb \& Conway, 2006; Fu \& Parks, 2001; Pascual, Pascual, Frías \& Rosel, 2006; Stevens, 1995; Varela, Prat, Voces \& Rial, 2006). En cambio, el sector de la alimentación y supermercados ha sido menos estudiado. Pascual et al. (2006) indican que la visión global que tiene el consumidor del establecimiento se basa en cuatro dimensiones: precio, producto, servicios/imagen y producto fresco.

Desde los años 60 hasta la actualidad, la definición de la satisfacción ha ido evolucionando de posiciones cognitivas a posiciones cognitivo-emocionales. Entre las evaluaciones cognitivas destacan aproximaciones a la satisfacción en términos de sensación de recompensa ante un esfuerzo (Howart \& Sheth, 1969), evaluación de la experiencia de compra (Hunt, 1977; Westbrook, 1980), evaluación de las expectativas iniciales y finales (Tse $\&$ Wilton, 1988), juicio experimental de la experiencia de consumo (Bolton \& Drew, 1991) y evaluación de la congruencia entre el resultado del acto de consumo y un estándar de comparación o referente, ya sean las expectativas, la experiencia (Erevelles \& Leavitt, 1992) o las necesidades y deseos (Kotler, 1991; Spreng \& Olshavsky, 1993). Dentro de los estándares de comparación, el modelo de confirmación de expectativas ha sido el más empleado (Martínez-Tur, Zurriaga, Luque \& Moliner, 2005; Marzo, Martínez-Tur, Ramos \& Peiró, 2002; Mira, Aranaz, Lorenzo, Rodríguez-Marín \& Moyano, 2001; Varela, Rial \& García, 2003) y el ajuste de las expectativas sería el factor crucial entre satisfacción y lealtad (Yi \& La, 2004).

Entre las posiciones cognitivo-emocionales destacan aproximaciones a la satisfacción en términos de evaluación cognitiva de unos atributos a la que suman la disposición emocional (Singh, 1991), respuesta afectiva derivada de la comparación de un resultado con un estándar anterior de compra o consumo (Halstead, Hartmon \& Schmidt, 1994), evaluación afectiva/cognitiva de la experiencia (Wirtz \& Bateson, 1994), estado cognitivo-afectivo resultante de la evaluación de las respuestas (Andreu, 2001) y proceso de acumulación (Terblanche, 
2006). Indiscutiblemente, los clientes experimentan emociones cuando interactúan con los empleados (Lee \& Dubinsky, 2003) y las variables ambientales del comercio también influyen sobre dichas emociones y, por lo tanto, en el comportamiento de compra (Gilboa \& Rafaeli, 2003), incluidos los elementos tangibles del ambiente (Varela et al., 2006).

Entre las consecuencias posibles más analizadas de la satisfacción está la lealtad. A nivel conductual, la lealtad se definiría como un comportamiento de los consumidores orientados hacia una marca, producto o tienda en el tiempo, operativizada a través de los patrones de repetición de compra o volumen de las mismas (Bustos \& González, 2006). No obstante, otros autores consideran insuficiente dicho enfoque para explicar el desarrollo y el mantenimiento de una verdadera lealtad (Dick \& Basu, 1994; Jacoby \& Kyner, 1973; Jones, Sasser \& Earl, 1995). Por otra parte, el concepto de lealtad no parece ser en absoluto monolítico. En este sentido, Yi (2004) postula seis tipos de lealtad diferentes: comportamental, actitudinal, cognitiva, afectiva, conativa y de acción. Estudios anteriores han demostrado que uno de los factores que influye en el hecho de volver a un establecimiento es la satisfacción (Bearden \& Teel, 1983; Gupta, McLaughlin \& Gómez, 2007; Szymansky \& Henard, 2001). Sin embargo, la satisfacción no es el único factor que guarda relación con la lealtad (Bigné \& Andreu, 2004), e incluso hay autores que ponen en duda dicha relación (Jones et al., 1995).

Otro aspecto por destacar en relación con el consumidor es el de los juicios cognitivos y afectivos que pueden derivarse de su experiencia, llevándole a que prevalezcan en él argumentos de compra funcionales o hedonistas (Anderson \& Weitz, 1992). El cliente predominantemente funcional se basa sobre todo en la confirmación de expectativas para satisfacer las necesidades, buscando argumentos más racionales como la cercanía, la comodidad, el tiempo de espera y/o de servicio, el precio, etc. Por el contrario, en el que priman los aspectos hedonistas basa principalmente la satisfacción personal en sus juicios, relacionándolos directamente con el placer, valorando más aspectos emocionales como la familiaridad, la personalización, etc. (Martínez-Tur et al., 2005). Rondán, Navarro y Diez (2007) abogan también por la segmentación de la población en distintos grupos de consumidores, que llevarán a diferentes tipos de comportamientos respecto al consumo, lo que influirá en la relación entre calidad percibida, satisfacción, confianza e intención de recompra.

El presente estudio tiene dos objetivos: a) revaluar la calidad percibida y los factores que influyen en constructos complejos y multidimensionales como la satisfacción y la fidelidad/lealtad en charcuterías y tiendas de delicatesen. Para la elaboración del instrumento se tomó como referencia el cuestionario propuesto por Varela et al. (2006), basado en la metodología SERVQUAL y se incluyeron dos preguntas sobre los porcentajes funcional (racional) y hedonista (placentero) que atribuía cada participante a su decisión de compra. b) Estudiar las variables que influyen en la satisfacción y la fidelidad/lealtad de los clientes en este ámbito. Se espera encontrar diferencias en función de las variables sociodemográficas consideradas, y además, que la satisfacción se relacione con la fidelidad/ lealtad. Debido a los pocos estudios que hay en este campo, se consideró adecuado focalizar la investigación en el pequeño comercio local, restringiendo el estudio exclusivamente al sector de charcuterías-delicatessen, dado que pueden ofrecer un componente hedonista mayor al de otro tipo de establecimientos.

\section{Método}

\section{Participantes}

La muestra estuvo formada por 712 participantes, 348 hombres y 364 mujeres, de 21 a 86 años de edad, que eran clientes habituales de charcuterías y tiendas de delicatessen de Cataluña. El 26\% pertenecía a la franja de 21-35 años, el 25.7\% tenía 36-50 años, el $23.7 \%$ 51-65 años y el $24.6 \% \geq 66$ años. El 18.1\% tenía un nivel socioeconómico bajo y medio-bajo, el $60.2 \%$ medio y el $21.7 \%$ medio-alto y alto. El 33.6\% tenía estudios superiores, el $34.1 \%$ secundarios y el $32.3 \%$ restante había cursado primaria o no tenía estudios. El 40.1\% informó que no trabajaba, mien- 
tras que el $37.4 \%$ lo hacía jornada completa y el $22.2 \%$ media jornada o jornada intensiva.

\section{Instrumentos}

El cuestionario final estaba formado por 34 enunciados, cuyo formato de respuesta era una escala Likert de 5 puntos (1: totalmente en desacuerdo a 5: totalmente de acuerdo). Las puntuaciones por escalas y para el total del cuestionario se calcularon a partir del promedio de los ítems correspondientes, previa recodificación de un ítem inverso. Además, el cuestionario incluía dos preguntas sobre los porcentajes funcional y hedonista que cada participante atribuía a su decisión de compra. La variable se operativizó sumando los dos valores y calculando qué porcentaje de dicha suma correspondía al componente hedonista.

\section{Procedimiento}

Se llevó a cabo un muestreo por cuotas de sexo y grupo de edad con afijación simple. El cuestionario se aplicó mediante entrevista personal estructurada, y los datos fueron recogidos durante abril de 2008.

\section{Análisis Estadísticos}

Los datos fueron analizados con SPSS v.19. Para verificar la estructura interna del cuestionario se aplicó un análisis en componentes principales con rotación VARIMAX. Para la extracción del número de componentes se tuvieron en cuenta los siguientes criterios: modelo teórico sobre la estructura de cinco dimensiones básicas (personal, servicios, producto, instalaciones y fidelidad), evidencias de otras posibles estructuras (entre 4 y 7 factores), criterio de Kaiser e interpretabilidad de la solución final. Para evaluar la fiabilidad de consistencia interna se calculó el alfa de Cronbach y el promedio de correlaciones inter-ítem. Las diferencias entre las diversas medidas de satisfacción, fidelidad y porcentaje de comportamiento hedonista en función de las variables demográficas sexo, edad, nivel socioeconómico, nivel de estudios y jornada se analizaron con $t$-Student para el factor sexo, ANOVA de un factor para la satisfacción global y MANOVA para las restantes medidas. La relación entre las puntuaciones de satisfacción y la puntuación de fidelidad se evaluó mediante regresión lineal múltiple.

\section{Resultados}

\section{Estructura interna y fiabilidad}

La prueba de adecuación muestral de Kaiser-Meyer-Olkin resultó satisfactoria $(\mathrm{KMO}=0.87)$ y la prueba de esfericidad de Bartlett fue estadísticamente significativa $\left(\chi_{(561)}^{2}=5025.9 ; p<0.001\right)$. La escala final propuesta para evaluar la calidad percibida incluye seis dimensiones que explican el $46.9 \%$ de la variancia total, cuyos valores propios (y \% de variancia explicada) tras la extracción fueron: 7.2 (21.3\%), 2.5 (7.2\%), 1.9 (5.5\%), 1.7 (5\%), $1.4(4.1 \%)$ y $1.3(3.9 \%)$. Las escalas resultantes tras la rotación son: trato-clientela (11.4\% de variancia explicada; 7 ítems), instalaciones (10.7\%; 7 ítems), producto $(8.5 \% ; 5$ ítems), servicios $(5.7 \% ; 7$ ítems), fidelidad (5.6\%; 4 ítems) y una nueva dimensión que denominamos "valor añadido" (5\%; 4 ítems). Solo algunos ítems han saturado en más de un factor, siendo estas saturaciones bajas (la mayoría entre 0.3 y 0.39 ) (Tabla 1 ).

La fiabilidad de consistencia interna resultó aceptable, atendiendo a la longitud de cada escala, con valores de alfa de Cronbach entre 0.46 (valor añadido) y 0.83 (trato-clientela). La correlación promedio inter-ítem resultó también adecuada para todas las escalas $(r \geq 0.2)$ excepto para la dimensión servicios $(r=0.12)$ y valor añadido $(r=0.19)$ (Tabla 1$)$.

\section{Variables sociodemográficas}

En la Tabla 2 se muestran las comparaciones de las puntuaciones en función de las variables sociodemográficas. No se hallaron diferencias en función del nivel socioeconómico y nivel de estudios, mientras que las mujeres mostraron una mayor satisfacción que los hombres en la puntuación global del cuestionario (IC95\%: [0.03; 0.16]) y las escalas instalaciones (IC95\%: [0.01; 0.17]), producto (IC95\%: 


\section{TABLA 1}

Resultados del análisis en componentes principales (rotación VARIMAX) y fiabilidad de consistencia interna de las escalas resultantes y del total del test

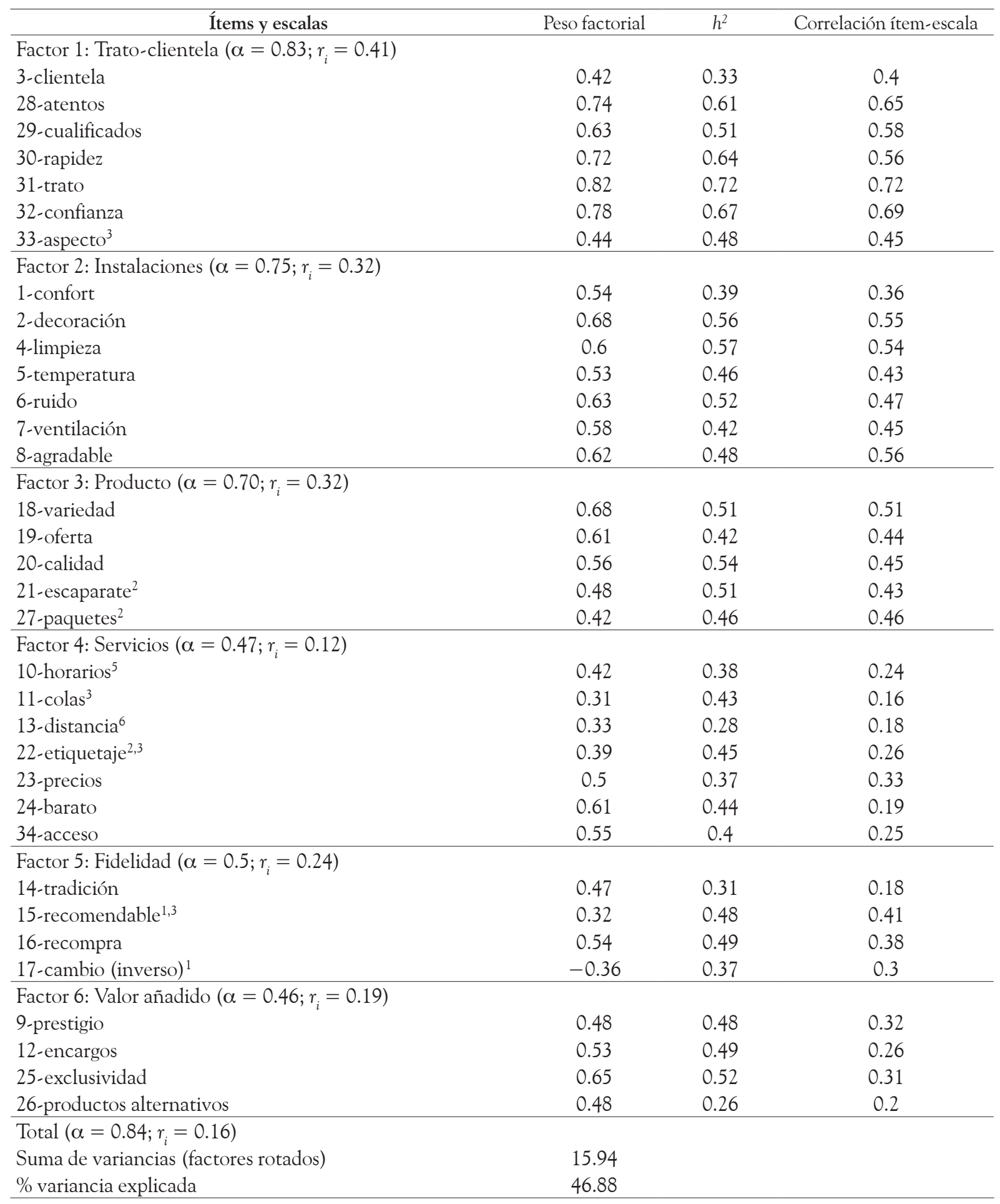

Nota. Los superíndices indican que el ítem satura también en el número de factor del superíndice (carga $\geq 0.3$ y diferencia $\leq$ 0.1 respecto al factor esperado). En cursiva: $h^{2}=$ comunalidad; $\alpha$ : alfa de Cronbach; $r_{i}$ : promedio de correlación inter-ítem. Fuente: elaboración propia 
[0.02; 0.19]), servicios (IC95\%: [0.01; 0.02]) y valor añadido (IC95\%: [0.01; 0.25]).

Los consumidores de 21 a 35 años mostraron una menor satisfacción con el trato-clientela (IC95\%: $[-0.38 ;-0.04] ;[-0.43 ; 0.08] ;[-0.5 ;-0.15]$ respectivamente), instalaciones (IC95\%: [-0.42; -0.06$]$ puntos con el grupo de más edad), producto (IC 95\%: [-0.38; -0.04]; [-0.44; 0.09] respectivamente), valor añadido (IC95\%:[ $-0.57 ;-0.09]$; $[-0.5$; 0.01] respectivamente) y satisfacción total (IC95\%: $[-0.29 ;-0.05] ;[-0.28 ; 0.04] ;[-0.36 ;-0.11]$ respectivamente), que alguno de los grupos de mayor edad, especialmente con el grupo de personas de más edad.

El grupo que no trabaja mostró mayor satisfacción global que el de jornada intensiva o media (IC95\%: [0.01; 0.22]) y mayor satisfacción de servicios que el de jornada completa (IC95\%: [0.03; 0.23]). La medida de hedonismo y las escalas de trato-clientela e instalaciones son casi-significativas.

En referencia a la fidelidad, solo se halló un mayor nivel de lealtad entre los consumidores con estudios superiores y los que solo tenían estudios primarios o no tenían estudios (IC95\%: [0.03; 0.35]). La diferencia entre los dos grupos extremos de edad también es significativa (IC95\%: [ -0.42 ; -0.01]). Asimismo, se encontraron diferencias entre los participantes que no trabajan y los que hacen media jornada o intensiva (IC95\%: [0.01; 0.35]).

\section{Relación entre Satisfacción y Fidelidad}

Las puntuaciones de satisfacción con el trato-clientela ( $\mathrm{B}=0.46$; IC95\%: $[0.37 ; 0.55] ; p<0.001)$, satisfacción con el producto $(B=1$; IC95\%: [0; 0.19]; $p=0.049)$ y la dimensión de valor añadido $(\mathrm{B}=$ 0.07; IC95\%: [0.01; 0.13]; $p=0.026$ ) se asociaron positivamente con la medida de lealtad, mientras que la satisfacción con las instalaciones y con los servicios no mostraron una relación significativa. El ajuste del modelo resultó aceptable $\left(R c^{2}=0.23\right)$.

\section{Discusión}

Ya al principio de este trabajo se justificó el interés por el estudio de la calidad, la satisfacción y su evaluación en el comercio local, en concreto, en el contexto de las charcuterías y tiendas de delicatessen, dada su indudable importancia en la economía y su carácter vitalizador. Este hecho, junto al gran número de establecimientos y a la fuerte competencia que existe, tanto entre ellos como respecto a las grandes superficies, hace que el tema de la calidad, la satisfacción y la fidelización sean cuestiones estratégicas y cruciales para el sector.

Por otra parte, el concepto de calidad y su evaluación es, asimismo, controvertido a nivel general (Martínez-Tur, Peiró \& Ramos, 2001; Varela et al., 2003), a lo que cabe añadir además la notable falta de trabajos empíricos en este sector. Adicionalmente, las variables sociodemográficas estudiadas desempeñan un papel importante, especialmente la edad y el sexo, tanto en la percepción como en la valoración de la calidad y la satisfacción. Por todo ello, se hace preciso elaborar instrumentos que permitan evaluar estos aspectos.

El primer objetivo ha sido una aproximación a la evaluación de la calidad percibida en estos establecimientos, con un cuestionario de 34 ítems que incluía seis dimensiones: trato-clientela, instalaciones, producto, servicios, fidelidad y valor añadido. Dado que se tomó como punto de partida el instrumento desarrollado por Varela et al. (2006) en el contexto de la hostelería, introduciendo algunas variaciones para adaptarlo al sector de la alimentación, se trata de una fase temprana del desarrollo y validación del instrumento y se decidió evaluar la estructura interna de forma exploratoria.

Los resultados indican unas puntuaciones globales positivas (alrededor de 4 en una escala de 5 puntos) y homogéneas ( $D E=0.4$ puntos). Entre las escalas destacan las puntuaciones más elevadas en trato-clientela, producto y fidelidad, mientras que servicios y valor añadido son las escalas con menor puntuación.

En cuanto a la importancia de cada uno de los factores sobre la explicación de la satisfacción, la dimensión trato-clientela es la que muestra una mayor relación, seguida a distancia por las instalaciones y el producto. Es poco relevante el rol del servicio, y la dimensión valor añadido se encuentra en un punto intermedio. Estos resultados podrían estar mediatizados por el hecho (común a la mayor 
TABLA 2

Media (y desviación estándar) de las diversas medidas del cuestionario segmentadas en función de las variables sociodemográficas consideradas y resultados de las comparaciones

\begin{tabular}{|c|c|c|c|c|c|c|c|c|}
\hline & $\begin{array}{c}\% \\
\text { Hedonismo }\end{array}$ & $\begin{array}{c}\text { Trato- } \\
\text { clientela }\end{array}$ & Instalaciones & Producto & Servicios & Fidelidad & $\begin{array}{c}\text { Valor } \\
\text { añadido }\end{array}$ & Total \\
\hline \multicolumn{9}{|l|}{ Sexo } \\
\hline Mujer & $29.1(20.9)$ & $4.2(0.6)$ & $3.9(0.6)^{*}$ & $4.2(0.6)^{*}$ & $3.7(0.5)^{*}$ & $4.1(0.7)$ & $3.7(0.8)^{*}$ & $4(0.4)^{*}$ \\
\hline Hombre & $29.7(22.1)$ & $4.2(0.6)$ & $3.8(0.6)^{*}$ & $4.1(0.6)^{*}$ & $3.6(0.5)^{*}$ & $4(0.7)$ & $3.6(0.8)^{*}$ & $3.9(0.4)^{*}$ \\
\hline$P$ prueba global & 0.697 & 0.095 & 0.044 & 0.017 & 0.032 & 0.094 & 0.029 & 0.002 \\
\hline \multicolumn{9}{|l|}{ Edad } \\
\hline 21-35 años & $30.2(21.9)$ & $4(0.6)^{a b c}$ & $3.7(0.6)^{\mathrm{d}}$ & $4(0.6)^{\mathrm{ef}}$ & $3.6(0.6)$ & $4(0.7)^{g}$ & $3.4(0.8)^{\mathrm{hi}}$ & $3.8(0.4)^{\mathrm{jkl}}$ \\
\hline 36-50 años & 29.9 (21.6) & $4.2(0.6)^{\mathrm{a}}$ & $3.9(0.6)$ & $4.2(0.6)^{\mathrm{e}}$ & $3.6(0.6)$ & $4(0.7)$ & $3.8(0.8)^{\mathrm{h}}$ & $4(0.4)^{j}$ \\
\hline 51-65 años & $30.4(21.2)$ & $4.3(0.6)^{b}$ & $3.8(0.6)$ & $4.2(0.6)$ & $3.6(0.5)$ & $4.1(0.7)$ & $3.7(0.8)^{\mathrm{i}}$ & $3.9(0.4)^{\mathrm{k}}$ \\
\hline 66 años o más & $27.3(21.3)$ & $4.3(0.5)^{c}$ & $4(0.6)^{d}$ & $4.3(0.5)^{f}$ & $3.7(0.5)$ & $4.2(0.7)^{g}$ & $3.7(0.8)$ & $4(0.4)^{1}$ \\
\hline$P$ prueba global & 0.524 & $<0.001$ & 0.003 & $<0.001$ & 0.108 & 0.047 & 0.001 & $<0.001$ \\
\hline \multicolumn{9}{|c|}{ Nivel socioeconómi } \\
\hline Bajo & $24(21.3)$ & $4.1(0.6)$ & $3.9(0.6)$ & $4(0.7)$ & $3.7(0.5)$ & $4.2(0.6)$ & $3.7(0.9)$ & $3.9(0.4)$ \\
\hline Bajo-medio & $27.5(20.8)$ & $4.2(0.6)$ & $3.7(0.6)$ & $4.1(0.6)$ & $3.7(0.6)$ & $3.9(0.7)$ & $3.5(0.8)$ & $3.8(0.4)$ \\
\hline Medio & $29.8(22.1)$ & $4.2(0.6)$ & $3.9(0.6)$ & $4.2(0.6)$ & $3.6(0.5)$ & $4.1(0.7)$ & $3.6(0.8)$ & $3.9(0.4)$ \\
\hline Medio-alto & $30(19.6)$ & $4.2(0.6)$ & $3.8(0.6)$ & $4.2(0.6)$ & $3.6(0.5)$ & $4(0.7)$ & $3.6(0.8)$ & $3.9(0.4)$ \\
\hline Alto & $35(30)$ & $4.5(0.8)$ & $3.9(0.6)$ & $4.2(1)$ & $3.9(0.9)$ & $4.1(0.9)$ & $3.7(0.8)$ & $4(0.6)$ \\
\hline$P$ prueba global & 0.617 & 0.608 & 0.126 & 0.334 & 0.654 & 0.175 & 0.85 & 0.385 \\
\hline \multicolumn{9}{|l|}{ Estudios } \\
\hline Primarios/sin & $27.7(21.5)$ & $4.3(0.6)$ & $3.9(0.6)$ & $4.2(0.6)$ & $3.6(0.5)$ & $4.2(0.7)^{\mathrm{m}}$ & $3.6(0.8)$ & $4(0.4)$ \\
\hline Secundarios & $29.8(20.4)$ & $4.2(0.6)$ & $3.9(0.6)$ & $4.2(0.6)$ & $3.6(0.6)$ & $4(0.7)$ & $3.7(0.8)$ & $3.9(0.4)$ \\
\hline Superiores & $30.5(22.1)$ & $4.1(0.6)$ & $3.8(0.6)$ & $4.1(0.6)$ & $3.6(0.5)$ & $4(0.7)^{\mathrm{m}}$ & $3.6(0.8)$ & $3.9(0.4)$ \\
\hline$P$ prueba global & 0.346 & 0.122 & 0.178 & 0.15 & 0.75 & 0.011 & 0.235 & 0.12 \\
\hline \multicolumn{9}{|l|}{ Jornada } \\
\hline Completa & $30.1(22)$ & $4.2(0.6)$ & $3.9(0.6)$ & $4.2(0.6)$ & $3.6(0.6)^{n}$ & $4(0.7)$ & $3.7(0.8)$ & $3.9(0.4)$ \\
\hline Intensiva/media & $32.1(22.1)$ & $4.1(0.6)$ & $3.7(0.6)$ & $4.1(0.7)$ & $3.6(0.6)$ & $3.9(0.7)^{\circ}$ & $3.6(0.8)$ & $3.8(0.5)^{\mathrm{p}}$ \\
\hline No trabaja & $27.3(20.6)$ & $4.3(0.5)$ & $3.9(0.6)$ & $4.2(0.6)$ & $3.7(0.5)^{\mathrm{n}}$ & $4.1(0.7)^{\circ}$ & $3.6(0.8)$ & $4(0.4)^{\mathrm{p}}$ \\
\hline$P$ prueba global & 0.07 & 0.071 & 0.065 & 0.121 & 0.021 & 0.038 & 0.154 & 0.024 \\
\hline
\end{tabular}

Nota. Los símbolos *,a,b,c,d,e,f,g,h,i,j,k,l,m,n,o,p indican los pares de comparaciones estadísticamente significativas en los contrastes a posteriori de Scheffé $(p<0.05)$.

Fuente: elaboración propia

parte de estudios de este tipo) que los participantes suelen ser clientes habituales del establecimiento, por lo que la variabilidad de los criterios se reduce drásticamente.

En cuanto al estudio de las diferencias en función de las variables sociodemográficas, no se hallaron diferencias en función del nivel socioeconómico y nivel de estudios, lo que parece corroborar que, independientemente de las características de los usuarios, todos los segmentos muestran un nivel de satisfacción similar y las puntuaciones son uniformemente elevadas. En futuros estudios proponemos la incorporación de una medida que evalúe si la persona es o no cliente habitual del establecimiento, con el fin de controlar este aspecto que ha mostrado estar relacionado en el sector de la hostelería con clientes de Cataluña y Bogotá (Berbel, 2011).

El hecho de que las mujeres muestren en general puntuaciones más elevadas podría atribuirse a que actualmente todavía son ellas las que realizan con mayor frecuencia las compras. Asimismo, el que los consumidores de menor edad (21-35 años) mostraran menor satisfacción también podría 
ser interpretado en este sentido. Ello sugiere que los grupos de edad pueden diferir debido a sus diferentes necesidades. Además, posiblemente la mayor utilización propicie un grado de satisfacción superior, por lo que, como se ha mencionado antes, dicho aspecto podría incorporarse en futuros estudios.

Por lo que respecta a la fidelidad, los consumidores con menor nivel de formación (estudios primarios o sin estudios) tuvieron puntuaciones más elevadas en lealtad que los participantes con estudios superiores; quizás un mayor nivel educativo favorezca un espíritu más crítico y estas personas decidan probar y comparar más opciones.

Por otra parte, el hecho que tres de las cinco medidas de satisfacción se asocien positivamente con la escala de fidelidad apoyaría la relación entre ambas variables (Gupta et al., 2007), aunque el ajuste del modelo indicaría que la satisfacción por sí sola es insuficiente para explicar el desarrollo y el mantenimiento de la lealtad (Dick \& Basu, 1994; Jacoby \& Kyner, 1973; Jones et al., 1995).

Por último, cabe señalar que la variable hedonismo no muestra diferencias entre los segmentos. Sus puntuaciones se encuentran en todos los casos alrededor de un 30\% de importancia del factor hedonista sobre la decisión de compra, lo que sin duda es un porcentaje importante, probablemente superior al que se encontraría en establecimientos de otro sector.

Este trabajo ha cubierto razonablemente los objetivos que pretendía, dado que el cuestionario tiene unas cualidades psicométricas satisfactorias y contribuye al desarrollo de este tipo de instrumentos, que permiten objetivar cuestiones tan relevantes como la satisfacción percibida y la fidelidad. Además, estos estudios proporcionan información a los propietarios de los establecimientos sobre cuáles son los aspectos que mejor y peor valoran los clientes, lo que ha de llevar potenciar los primeros y la mejora de los más deficitarios. En futuros estudios sería interesante extender la aplicación (y adaptación) del cuestionario a otros tipos de comercio local con el fin de conocer si los resultados son similares.

\section{Referencias}

Andaleeb, S. S., \& Conway, C. (2006). Customer satisfaction in the restaurant industry: An examination of the transaction-specific model. Journal of Services Marketing, 20(1), 3-11.

Andreu, L. (2001). Emociones y satisfacción del consumidor. Propuesta de un modelo cognitivo-afectivo en servicios de ocio y turismo. Tesis Doctoral, Universidad de Valencia, España.

Bearden, W. O., \& Teel, J. E. (2004). Selected determinants of consumer satisfaction and complaints reports. Journal of Marketing Research, 20(1), 21-28.

Berbel, G. (2011). Anàlisi metodològica multinivell de la satisfacció en restaurants. Tesis Doctoral, Universitat Autònoma de Barcelona, España.

Bigné, J., \& Andreu, L. (2004). Emotions in segmentation: An empirical study. Annals of Tourism Research, 31(3), 682-696.

Bolton, R. N., \& Drew, J. (1991). A multistage model of customers' assessments of service quality and value. Journal of Consumer Research, 17(4), 375-384.

Bustos, C., \& González, O. (2006). Papel del formato comercial en la lealtad al establecimiento minorista. Información Comercial Española. Revista de Economía, 828, 269-288.

Dick, A. S., \& Basu, K. (1994). Customer loyalty: Toward an integrated conceptual framework. Journal of the Academy of Marketing Science, 22(2), 99-113.

Erevelles, S., \& Leavitt, C. (1992). A comparison of current models of consumer satisfaction/dissatisfaction. Journal of Consumer Satisfaction, Dissatisfaction and Complaining Behavior, 5(1), 104-114.

Fu, Y. Y., \& Parks, S. C. (2001). The relationship between restaurant service quality and consumer loyalty among the elderly. Journal of Hospitality $\mathbb{E}$ Tourism Research, 25(3), 320-326.

Gilboa, S., \& Rafaeli, A. (2003). Store environment, emotions and approach behaviour: Applying environmental aesthetics to retailing. The International Review of Retail, Distribution \& Consumer Research, 13(2), 195-211.

Gupta, S., McLaughlin, E., \& Gómez, M. (2007). Guest satisfaction and restaurant performance. Cornell Hotel $\mathbb{E}$ Restaurant Administration Quarterly, 48(3), 284-298. 
Halstead, D., Hartman, D., \& Schmidt, S.L. (1994). Multisource effects on the satisfaction formation process. Journal of the Academy of Marketing Science, 22(2), 114-129.

Howard, J. A., \& Sheth, J.N. (1969). The theory of buyer behavior. New York: John Wiley \& Sons.

Hunt, H. K. (1977). CS/D: Overview and Future Research Directions. En H. K. Hunt. (Ed.), Conceptualization and measurement of consumer satisfaction and dissatisfaction (pp. 455-488). Cambridge, MA: Marketing Science Institute.

Jacoby, J., \& Kyner, D. (1973). Brand loyalty vs. repeat purchasing behaviour. Journal of Marketing Research, 10(1), 1-9.

Johnson, M. D., Herrmann, A., \& Gustafson, A. (2002). Comparing customer satisfaction across industries and countries. Journal of Economic Psychology, 23(6), 749-769.

Jones, T., Sasser, W., \& Earl, W., Jr. (1995). Why satisfied customers defect. Harvard Business Review, 73(6), 88-99.

Kotler, P. (1991). Marketing management (7.a ed.). Englewood Cliffs, NJ: Prentice Hall.

Lee, S., \& Dubinsky, A. (2003). Influence of salesperson characteristics and customer emotion on retail dyadic relationships. The International Review of Retail, Distribution and Consumer Research, 13(12), 21-36.

Martínez-Tur, V., Peiró, J. M., \& Ramos, J. (2001). Calidad de servicio y satisfacción del cliente. Madrid: Síntesis.

Martínez-Tur, V., Zurriaga, R., Luque, O., \& Moliner, C. (2005). Efecto modulador del tipo de segmento en la predicción de la satisfacción del consumidor. Psicothema, 17(2), 281-285.

Marzo, J., Martínez-Tur, V., Ramos, J., \& Peiró, J. (2002). La satisfacción del usuario desde el modelo de la confirmación de expectativas: Respuesta a algunos interrogantes. Psicothema, 14(4), 765-770.

Mira, J. J., Aranaz, J., Lorenzo, S., Rodríguez-Marín, J., \& Moyano, S. (2001). Evolución de la calidad percibida por los pacientes en dos hospitales públicos. Psicothema, 13(4), 581-585.

Parasuraman, A., Zeithaml, V. A., \& Berry, L. L. (1988). SERVQUAL: A multiple-item scale for measuring consumer perceptions of service quality. Journal of Retailing, 64(1), 12-40.

Parasuraman, A., Zeithaml, V. A., \& Berry, L. L. (1991). Refinement and reassessment of the SERVQUAL Scale. Journal of Retailing, 67(4), 420-450.

Pascual, M., Pascual, J., Frías, M. D., \& Rosel, J. (2006). Calidad de servicio en supermercados: una propuesta de medición. Psicothema, 18(3), 661-667.

Rondán, F., Navarro, A., \& Diez, E. (2007). Proposing new variables for the identification of strategic groups in franchising. International Entrepreneurship and Management Journal, 3(4), 355-377.

Singh, J. (1991). Understanding the structure of consumers' satisfaction evaluations of service delivery. Journal of the Academy of Marketing Science, 19(3), 223-245.

Spreng, R. A. \& Olshavsky, R. W. (1993). A desires congruency model of consumer satisfaction. Journal of the Academy of Marketing Science, 21(3), 169-177.

Stevens, P. (1995). DINESERV: A tool for measuring service quality in restaurants. Cornell Hotel $\mathcal{E}^{2}$ Restaurant Administration Quarterly, 36(2), 56-60.

Szymanski, D. M., \& Henard, D. D. (2001). Customer satisfaction: A meta-analysis of the empirical evidence. Journal of the Academy of Marketing Science, 29(1), 16-35.

Terblanche, N. S. (2006). An application of the American Customer Satisfaction Index (ACSI) in the South African motor vehicle industry. South African Journal of Business Management, 37(4), 29-38.

Tse, D. K., \& Wilton, P. C. (1988). Models of consumer satisfaction formation: An extension. Journal of Marketing Research, 25(2), 204-212.

Varela, J., Prat, R., Voces, C., \& Rial, A. (2006). Una nueva escala para la evaluación de la calidad de los servicios de hostelería. Psicothema, 18(1), 135-142.

Varela, J., Rial, A., \& García, E. (2003). Presentación de una escala de satisfacción con los servicios sanitarios de atención primaria. Psicothema, 15(4), 656-661.

Westbrook, R. A. (1980). Intrapersonal affective influences on consumer satisfaction with products. Journal of Consumer Research, 7(1), 49-54.

Wirtz, J., \& Bateson, J. E. (1999). Consumer satisfaction with services: Integrating the environment perspective in services marketing into the traditional 
disconfirmation paradigm. Journal of Business Research, 44(1), 55-66.

Yi, Y. (2004). Service marketing (3.a ed.). Seoul: Hankhyunsa.
Yi, Y., \& La, S. (2004). What influences the relationship between customer satisfaction and repurchase intention? Investigating the effects of adjusted expectations and customer loyalty. Psychology $\mathbb{E}$ Marketing, 21(5), 351-373. 